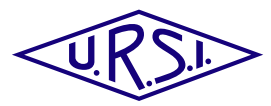

\title{
Revealing the geometry of gravitational wave event GW 170817 with radio observations
}

\author{
Poonam Chandra ${ }^{(1)}$, Kunal Mooley ${ }^{(2)}$, Dale Frail ${ }^{(3)}$, Gregg Hallinan ${ }^{(2)}$ and Kishalay De $^{(2)}$ \\ (1) National Centre for Radio Astrophysics, Tata Institute of Fundamental Research, Pune, 411007, India \\ (2) Caltech, 1200 E. California Blvd. MC 249-17, Pasadena, CA 91125, USA \\ (3) National Radio Astronomy Observatory, Socorro, New Mexico 87801, USA
}

GW 170817 marks the first gravitational wave detection of a binary neutron-star (BNS) merger by the Advanced LIGO and the Advanced VIRGO detectors. This is also the first gravitational wave event from which the electromagnetic (EM) emission was seen all the way from gamma-rays to radio bands. We detected it in radio bands 16 days after the detection [2].

With the detection of a short gamma ray burst by the Fermi after a delay of $1.7 \mathrm{~s}$, at first it seemed to confirm the association of short gamma ray bursts with the BNS mergers. However, the the peculiar characteristics of the gamma-ray emission and the radio data till first one hundred days showed inconsistencies with this traditional model. With the rich and well sampled radio data using multiple radio telescopes such as JVLA, ATCA and uGMRT, combined with the Very Long Baseline Interferrometry measurements, we have been able to understand the geometry and energetics of this exciting event [1].

The journey of this special event shows how the radio observations have played a crucial role in unfolding the very nature of this event. Our radio observations have suggested the initial radio emission to be from the cocoon and later the 20 degrees off-axis jet started to dominate the emission.

GW170817 represents only an initial exploration of a rich scientific landscape populated merger of massive binary systems. The study of radio afterglows of neutron star mergers detected by LIGO/Virgo in their upcoming observing runs will be able to address many unsolved questions in this field.

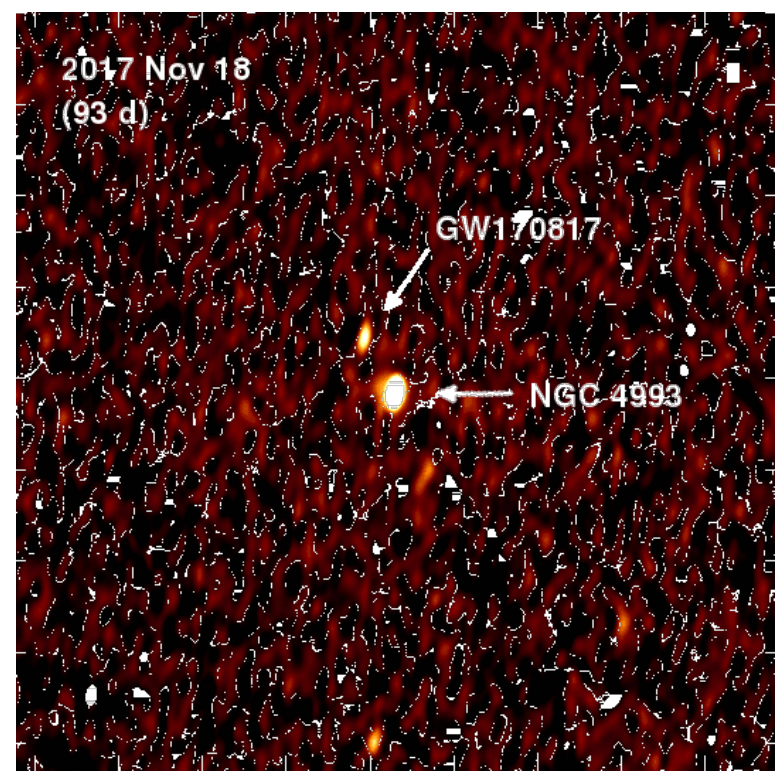

Figure 1. Radio detection of teh GW 170817 event with the VLA [2].

\section{References}

[1] Mooley, K. P.; Frail, D. A.; Dobie, D. et al., "A Strong Jet Signature in the Late-time Light Curve of GW170817,” The Astrophysical Journal Letters, 868, 2018, pp. 11-19, doi:10.3847/2041-8213/aaeda7.

[2] Hallinan, G.; Corsi, A.; Mooley, K. P et al., "A radio counterpart to a neutron star merger," Science, 358, 2017, pp. 1579-1583, doi: 10.1126/science.aap9855. 